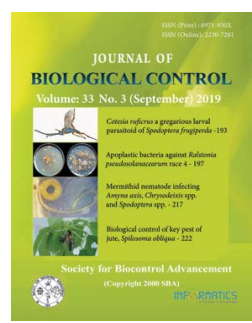

Research Article

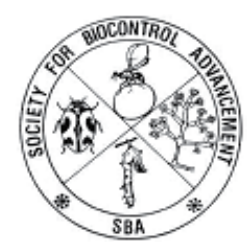

\title{
Diversity of cry genes occurring in the North East
}

\author{
R. RANGESHWARAN ${ }^{1 *}$, V. VELAVAN ${ }^{2}$, SATENDRA KUMAR ${ }^{1}$, V. APOORVA ${ }^{1}$, K. M. VENUGOPALA ${ }^{1}$, A. N. \\ SHYLESHA ${ }^{1}$ and G. SIVAKUMAR ${ }^{1}$ \\ ${ }^{1}$ ICAR-National Bureau of Agricultural Insect Resources, H.A. Farm Post, Hebbal, Bellary Road, Bengaluru - 560024, \\ Karnataka, India \\ ${ }^{2} I C A R$-National Research Centre for Banana, Thogaimalai Rd, Podavur, Thiruchirapalli-620102, Tamil Nadu, India \\ *Corresponding author E-mail: rangeshw@gmail.com
}

\begin{abstract}
The search for new Bacillus thuringiensis (Bt) strains is a continuous process and researchers are now focusing on finding toxin proteins that are toxic to pests of insect orders that are not reported. In the present study soil and insect cadaver samples were collected from North East India comprising the states of Assam, Tripura and Mehhalaya and native Bt were isolated using standard protocols. At total of $30 \mathrm{Bt}$ isolates were purified and characterized. Various types of crystal morphology were encountered that included bipyramidal, cuboidal, square, rhomboid, spherical and irregular. PCR analysis showed that diverse $c r y$ genes were expressed. The $c r y$ genes identified were Lepidoptera, Coleoptera and Diptera specific. Detected genes included cry1Ac, cry2A, cry4A, cry10A, cry16A, cry17A, cry19A, cry30Aa, cry $44 A a$, cry $11 A, c r y 4 B, c r y 12 A$, cry $8 A$ and cry $7 A$. Many of them were positive for Vip3A protein. The coleopteran specific $B t$ were evaluated against Sitophilus oryzae and Callosobruchus chinensis and NBAIR-AgBt6 was found to be toxic. The isolates are being further evaluated for use as biopesticides.
\end{abstract}

KEY WORDS: Bacillus thuringiensis, bioassay, cry genes, diversity, North East

(Article chronicle: Received: 16-07-2018; Revised: 12-08-2019; Accepted: 25-08-2019)

\section{INTRODUCTION}

The search for new Bacillus thuringiensis (Bt) strains is a continuous process and researchers are now focusing on finding toxin proteins that are toxic to pests of insect orders that are not reported. Basically search for novel Cry toxins is the norm. However studies on $B t$ diversity are well documented from different countries (Bravo et al., 1998; Porcar and Juarez Perez, 2003; Uribe et al., 2003; Quesada Moraga et al., 2004; Nazarian et al., 2009; Thammasittirong and Attathom, 2008; Baig and Mehnaz, 2009). In India limited work on $B t$ diversity has been done (Ramalakshmi and Udayasuriyan, 2010; Asokan et al., 2014) but the studies were restricted to certain Cry types like $c r y 3$ or $c r y 1$. Improved PCR based techniques have been designed to detect cry genes (Bravo et al., 1998; Beron et al., 2005). Documentation of Bt diversity in northeast India is very limited or not available. Currently there are 240 holotype toxins available (www. lifesci.sussex.ac.uk). Under cryl there are 44 types like crylAa, crylAb, crylAc etc, similarly under cry 2 there are 9 types like $c r y 2 A a$, cry $2 A b$ etc. We have toxins listed from cry1 to cry 72 (having 229 holotypes). Hence we undertook a systematic study to analyse the type of $B t$ that occur in northeast India (Tripura, Assam and Shillong) and also to ascertain the cry gene diversity that occurs in these isolates. The strategy used was based on multiplex PCR analysis with novel, general and specific primers for identification of Cry toxin genes for lepidopteran, coleopteran and dipteran pests.

\section{MATERIALS AND METHODS}

\section{Isolationof Bacillus thuringiensiss strains from soil samples and insect cadavers}

Soil and insect cadaver samples were collected from the three states of northeast India (Tripura, Assam and Meghalaya). Samples were drawn from forests and crop growing areas. It was ascertained that commercial $B t$ formulation was not used in these areas. The $B t$ isolates were selected as per the methodology of Travers et al. (1987) and Santana et al. (2008).

\section{Staining and microscope analysis}

The $B t$ isolates were screened for production of crystalline inclusions by use of amido black stain. The stain was prepared as A. $1.5 \mathrm{~g}$ amido black stain $10 \mathrm{~b}$ dissolved in $1 \mathrm{~L}$ containing $50 \%$ methanol, $40 \%$ distilled water, $10 \%$ 
glacial acetic acid, filtered and stored for 3 days; and B. 0.5\% aqueous solution of safranin. Heat fixed smear was stained with A for 2-3 minutes, washed and stained with B for 2-3 minutes, washed, dried and observed under oil immersion using Olympus BX-41. Crystals appeared blue black and spores appeared with pinkish margin. Crystals were also characterized by Transmission Electron Microscopy (TEM) studies by uranyl acetate staining and observation in TEM make HITACHI.

\section{PCR amplification and detection of cry genes}

The $B t$ isolates that produced protein crystals were purified. The purified cultures were grown overnight in LB broth in rotary shaker (model Orbitek) at $250 \mathrm{rpm}$. DNA was extracted by using HiPurA ${ }^{\mathrm{TM}}$ Bacterial Genomic Purification Kit (MB505) as per the manufacturer's protocol with miniprep column formation as per Sambrook (2001). Purity of DNA was tested by running in $1.25 \%$ agarose gel for $1 \mathrm{hr}$ at 100V. Multiple cry gene profile in PCR was done using Quantarus (UK) make thermocycler for 30 reaction cycles each. PCR reactions were carried out in $25 \mu \mathrm{l}$ containing 20100ng of DNA mixed with 1X Taq reaction buffer, $150 \mathrm{mM}$ - dNTP mix, 0.2-0.4 $\mu \mathrm{M}$ - Primer (forward and reverse), $1 \mathrm{U}$ Taq DNA Polymerase (GeNei), $14 \mu \mathrm{M}-\mathrm{MgCl}_{2}$. Denaturation of template DNA was done for one minute at $94^{\circ} \mathrm{C}$, annealing at $45-59^{\circ} \mathrm{C}$ (Table 1) for one minute and elongation at $72^{\circ} \mathrm{C}$. An extra denaturation and elongation step was provided at $94^{\circ} \mathrm{C}$ for $2-5$ minutes and at $72^{\circ} \mathrm{C}$ for $5-10$ minutes respectively (Aly, 2007). PCR amplification of products were separated using agarose gel electrophoresis in $1.2 \%$ TAE buffer and stained with $0.2 \mathrm{mg} / \mathrm{ml}$ ethidium bromide (Sambrook et al., 1989). PCR products were visualized under UV transilluminator and the sizes of the fragments were estimated based on a DNA ladder (GeNei) of 100 to 1500 base pairs. The specific primers used are listed in (Table 1). For Vip3A specific primers were designed by us.

\section{Preparation of Bacillus thuringiensis extracts for bioassays}

Bacillus thuringiensis $24 \mathrm{~h}$ overnight culture in single colony of each strain grown in T3 agar medium were inoculated in $10 \mathrm{ml}$ liquid LB broth medium and grown for $48 \mathrm{~h}$ at $28^{\circ} \mathrm{C}$ and $200 \mathrm{rpm}$. An aliquot was taken to verify spore and crystal formation (over $90 \%$ sporulation is optimum), and the pre-culture was incubated for $20 \mathrm{~min}$ at $70^{\circ} \mathrm{C}$ to eliminate vegetative cells (synchronization). The main culture $(40 \mathrm{ml})$ was inoculated with $1 / 1,000$ volumes of synchronized pre-culture and grown as mentioned above. Optimal crystal formation was checked by phase-contrast microscopy. The whole culture was centrifuged at $9,000 \times \mathrm{g}$ for $10 \mathrm{~min}$. An aliquot of the supernatant $(1 \mathrm{ml})$ was kept at $-20^{\circ} \mathrm{C}$ for future bioassays. The pellet was washed once with ice-cold $1 \mathrm{~mol} / 1 \mathrm{NaCl}, 10 \mathrm{mmol} / \mathrm{l}$ EDTA solutions. Finally, the pellet was suspended in $1 \mathrm{ml}$ of $10 \mathrm{mmol} / \mathrm{KCl}$. OD $590 \mathrm{~nm}$ was measured and suspensions were stored at $-20^{\circ} \mathrm{C}$ until bioassay. All steps after centrifugation were done on ice to limit proteolysis.

\section{Protein estimation}

The pellet obtained from above was, dissolved in $500 \mu 1$ of lysis buffer containing $100 \mathrm{mM}$ Tris $\mathrm{HCl}$ (pH 7.0), $20 \mathrm{mM}$ EDTA, $5 \mathrm{mg} / \mathrm{ml}$ lysozyme, $2 \%$ SDS and it was centrifuged at $8000 \mathrm{rpm}$ for 7 minutes. Pellet was resuspended in $200 \mu 1$ resuspension buffer $(0.1 \%$ SDS $+10 \mathrm{mM}$ EDTA $)$, further diluted with treatment buffer $(2.0 \% \operatorname{SDS}+5 \%$ â mercaptoethanol $+130 \mathrm{mM}$ Tris $\mathrm{HCl}, \mathrm{pH} 10.0)$ and incubated at $90^{\circ} \mathrm{C}$ for 7 minutes (Morris et al., 1998). Protein concentration was measured as per Lowry et al. (1951).

\section{Bioassay}

Sitophilus oryzae and Callosobrochus chinensis were obtained from the division of insect ecology that were maintained as stored grain pests on chickpea seeds. The beetles were reared in the laboratory at $28-32{ }^{\circ} \mathrm{C}$ and $70-80 \%$ of relative humidity on maize and chickpea grains. The spore crystal protein concentrations obtained from the $B t$ that expressed coleopteran specific toxin genes were serially diluted to obtain six different concentrations. Two types of grains were used, for $C$. chinensis chickpea groundnut seeds were used and for $S$. oryzae maize seeds were used. The fully grown seeds (100 numbers) were first disinfested $\left(65^{\circ} \mathrm{C}\right.$ for $\left.2-3 \mathrm{~h}\right)$, cooled and then surface coated with the $2 \mathrm{ml}$ of different protein (spore crystal) concentration with 3 replications for each treatment. The seeds were then transferred into Petri dishes. For each treatment ten healthy laboratory reared beetles were exposed with help of soft brush. For control both dry grains and wet (sterile water treated) grains with three replicas were maintained. Mortality was recorded on daily basis separating dead from alive and average of three replicas were considered as final mortality (Md. Abdur Rashid et al., 2012). The results were subjected to probit analysis using SPSS version 10 software and $\mathrm{LC}_{50}$ values obtained.

\section{RESULTS AND DISCUSSION}

Thirty Bacillus thuringiensis bacteria were isolated from a total of 123 samples including soil, and dead insects obtained from North East encompassing three states (Assam, 
Table 1. List of primers used for the detection of different cry genes from North-East isolates of Bacillus thuringiensis

\begin{tabular}{|c|c|c|c|c|}
\hline Cry gene & $\begin{array}{l}\text { Product } \\
\text { size (bp) }\end{array}$ & Primers sequence & $\begin{array}{l}\text { Annealing } \\
\text { Temp. }\left({ }^{\circ} \mathrm{C}\right)\end{array}$ & Reference \\
\hline Cry $1 A c$ & 3686 & $\begin{array}{l}\text { P1 5'GTCGACTATGGATAACAATCCG'3 } \\
\text { P2 5'-GGCTCCDDAACCTGAGTTTGC-3' }\end{array}$ & 58 & Designed in this experiment \\
\hline $\operatorname{Cry} 2 A$ & 1170 & $\begin{array}{l}\text { 5'-CGATATGTTAGAATTTAGAAC-3' } \\
\text { 5'-TACCGTTTATAGTAACTCG-3' }\end{array}$ & 50 & Porcar and Perez 2003 \\
\hline Cry $3 A$ & 951 & $\begin{array}{l}\text { 5'-CGTTATCGCAGAGAGATGACATTAAC-3' } \\
\text { 5'-TGGTGCCCCGTCTAAACTGAGTGT-3' }\end{array}$ & 59 & Ben Dov et al., 1997 \\
\hline Cry $4 A$ & 1529 & $\begin{array}{l}\text { 5'-GGGTATGGCACTCAACCCCACTT-3', } \\
\text { 5'-GCGTGACATACCCATTTCCAGGTCC-3' }\end{array}$ & 53 & Ben Dov et al., 1997 \\
\hline Cry $4 B$ & 1925 & $\begin{array}{l}\text { 5'-GGGTATGGCACTCAACCCCACTT-3' } \\
\text { 5'-GCGTGACATACCCATTTCCAGGTCC-3' }\end{array}$ & 55.9 & Ben Dov et al., 1997 \\
\hline Cry $7 A$ & 1320 & $\begin{array}{l}\text { 5'-CATCTAGCTTTATTAAGAGATTC-3' } \\
\text { 5'-GATAAATTCGATTGAATCTAC-3' }\end{array}$ & 59.2 & Ben Dov et al., 2001 \\
\hline Cry $8 A$ & 342 & $\begin{array}{l}\text { 5'-ATGAGTCCAAATAATCTAAATG-3' } \\
\text { 5'-TCTCCCCATATATCTACGCTC-3' }\end{array}$ & 50 & Bravo et al., 1998 \\
\hline Cry $10 A$ & 651 & $\begin{array}{l}\text { 5'-ATAAATTCAAGTGCCAAGTA-3' } \\
\text { 5'-CCGAACCTACTATTGCGCCA-3' }\end{array}$ & 45 & Porcar et al., 1999 \\
\hline Cry $11 \mathrm{~A}$ & 445 & $\begin{array}{l}\text { 5'-CCGAACCTACTATTGCGCCA-3' } \\
\text { 5'-CTCCCTGCTAGGATTCCGTC-3' }\end{array}$ & 55 & Ben Dov et al, 1997 \\
\hline Cry $12 \mathrm{~A}$ & 363 & $\begin{array}{l}\text { 5'-CTCCCCCAACATTCCATCC-3' } \\
\text { 5'-AATTACTTACACGTGCCATACCTG-3' }\end{array}$ & 59.3 & Ejiofor A.O. and Johnson T. (2002) \\
\hline Cry $16 \mathrm{~A}$ & 1415 & $\begin{array}{l}\text { 5'-TCAAAAGGTGTGGCAAG-3' } \\
\text { 5'-ATAAGCCCAATATCATG-3' }\end{array}$ & 46 & Barloy et al., 1998 \\
\hline Cry $17 A$ & 1400 & $\begin{array}{l}\text { 5'-AAGTAAAGATTTCTGGG-3' } \\
\text { 5'-CTGAGGTATTTTGTGGA-3' }\end{array}$ & 48 & Barloy et al., 1998 \\
\hline Cry $19 A$ & 355 & $\begin{array}{l}\text { 5'-AGGGGAGTCCAGGTTATGAGTTAC-3' } \\
\text { 5'-ATTTCCCTAGTTAGTTCGGTTTTT-3' }\end{array}$ & 46.9 & Ejiofor A.O. and Johnson T. (2002) \\
\hline Cry $30 A a$ & 1600 & $\begin{array}{l}\text { 5'-ACAAATTATAAAGATTGGCT-3' } \\
\text { 5'-GAGTAATTGGCAGAAATTC-3' }\end{array}$ & 52 & Ito et al., 2006 \\
\hline Cry $44 A a$ & 1800 & $\begin{array}{l}\text { 5'-ACAAATT ATAAAGATTGGCT-3' } \\
\text { 5'-GAGTAATTGGCAGAAATTC-3' }\end{array}$ & 53 & Ito et al., 2006 \\
\hline Vip3A & 2370 & $\begin{array}{l}\text { F 5' CTC AAT GGG ACG CAT TTC TT 3' } \\
\text { R } 5 \text { 'GTTGTAAGGGCACTGTTC 3' }\end{array}$ & 50 & Rangeshwaran et al., 2016 \\
\hline
\end{tabular}

Tripura and Shillong). The crystal structures of these isolates were identified using Amido black staining. The isolates expressed varied type of crystals (bypramidal, square, spherical, irregular) as observed through transmission electron microscopy (Fig. 1).

The cry gene profiling of the $30 B t$ isolates from Northeast was done by amplification of specific cry genes using degenerate primers, PCR amplification and sequence analysis (Table 2). Here 16 types of cry genes were detected from the 30 isolates. Some of them were sequenced and submitted to GenBank. The amplified Cry protein genes included CrylAc, Cry2A, Cry3A, Cry4A, Cry4B, Cry7A, Cry8A, Cry10A, Cry11A, Cry12A, Cry16A, Cry17A, Cry19A, Cry30Aa,
Cry44A $a$ and Vip3A (Table 2 and 3). All the isolates harboured genes that target pests of more than one insect order. For example ten strains namely TrBt10, TrBt17, AsBT21, AsBT20, AsBT24, TrBt8, TrBt10, AsBt16, TrBt18 and AsBt16 harboured cry genes that are Lepidoptera-Diptera-Coleoptera-active. Groups or combination Cry protein genes detected against insect orders were Lepidoptera; Coleoptera, Diptera; Lepidoptera-Diptera; Lepidoptera-Coleoptera; Coleoptera-Dipteraand LepidopteraDiptera-Coleoptera. Vip3Aprotein gene amplification was found in 17 of the 30 North-East isolates. Cry $1 A c$ and $C r y 2 A$ invariably occurred together in all the isolates (Table 2). Hence LepidopteraDiptera active cry genes were abundant. One unique isolate TrBt-18 which showed bipyramidal and spherical crystals harboured $C r y 2 A, C r y 4 A, C r y 7 A, C r y 8 A, C r y 16 A$, and Vip3A 
Table 2. Cry gene profiles of the Bacillus thuringiensis isolates from North East

\begin{tabular}{|c|c|c|c|c|c|c|}
\hline $\begin{array}{l}\text { Strain } \\
\text { Name }\end{array}$ & $\begin{array}{c}\text { Crystal } \\
\text { Morphology }\end{array}$ & Cry gene profile & $\begin{array}{l}\text { Isolation } \\
\text { source }\end{array}$ & GPS data & $\begin{array}{l}\text { Accession } \\
\text { No }\end{array}$ & $\begin{array}{l}\text { Predicted insecticidal } \\
\text { activity }\end{array}$ \\
\hline AgBt-1 & $\begin{array}{l}\text { Bipyramidal }+ \\
\text { cuboidal }\end{array}$ & $\begin{array}{c}\text { CrylAc, Cry2A, Cry10A, } \\
\text { Cry16A, Cry17A, Cry19A, } \\
\text { Cry30Aa, Cry } 44 A a \text { and Vip3A }\end{array}$ & $\begin{array}{l}\text { Adult } \\
\text { larvae }\end{array}$ & $23^{\circ} 51^{\prime} \mathrm{N} 91^{\circ} 16^{\prime} \mathrm{E}$ & & Lepidoptera+Diptera \\
\hline AgBt-2 & $\begin{array}{l}\text { Bipyramidal }+ \\
\text { spherical }\end{array}$ & Cry $1 A c$ & Pupae & $23^{\circ} 51^{\prime} \mathrm{N} 91^{\circ} 16^{\prime} \mathrm{E}$ & & Lepidoptera \\
\hline AgBt-3 & Bipyramidal & $\begin{array}{c}\text { Cry1Ac, Cry16A, Cry17A, } \\
\text { Cry19A, Cry 30Aa, Cry44Aa } \\
\text { and Vip3A }\end{array}$ & Leaf & $23^{\circ} 51^{\prime} \mathrm{N} 91^{\circ} 16^{\prime} \mathrm{E}$ & & Lepidoptera + Diptera \\
\hline AgBt-4 & $\begin{array}{l}\text { Bipyramidal + } \\
\text { Irregular } \\
\end{array}$ & CrylAc, Cryl1A and vip3A & $\begin{array}{l}\text { Dead } \\
\text { larvae }\end{array}$ & $23^{\circ} 51^{\prime} \mathrm{N} 91^{\circ} 16^{\prime} \mathrm{E}$ & KC596019 & Lepidoptera + Diptera \\
\hline AgBt-5 & Bipyramidal & $\begin{array}{c}\text { CrylAc, Cry2A, Cry } 4 A, C r y 10 A, \\
\text { Cry11A, Cry16A, Cry19A and } \\
\text { Vip3A }\end{array}$ & $\begin{array}{l}\text { Dead } \\
\text { larvae }\end{array}$ & $23^{\circ} 51^{\prime} \mathrm{N} 91^{\circ} 16^{\prime} \mathrm{E}$ & & Lepidoptera + Diptera \\
\hline AgBt-6 & $\begin{array}{l}\text { Bipyramidal }+ \\
\text { irregular }\end{array}$ & $\begin{array}{c}\text { CrylAc, Cry2A, Cry } 4 A, \text { Cry10A, } \\
\text { Cry11A, Cry16A, Cry19A, } \\
\text { Cry } 44 A a \text { and Vip3A }\end{array}$ & $\begin{array}{l}\text { Dead } \\
\text { larvae }\end{array}$ & $24^{\circ} 32^{\prime} \mathrm{N} 92^{\circ} 21^{\prime} \mathrm{E}$ & KC596018 & Lepidoptera + Diptera \\
\hline AgBt-7 & $\begin{array}{l}\text { Bipyramidal } \\
\text { crystal }\end{array}$ & Cry1Ac, Cry16A, Cry19A & Leaf & $24^{\circ} 32^{\prime} \mathrm{N} 92^{\circ} 21^{\prime} \mathrm{E}$ & & Lepidoptera + Diptera \\
\hline TrBt-8 & $\begin{array}{l}\text { Bipyramidal } \\
\text { crystal }\end{array}$ & $\begin{array}{c}C r y 4 A, C r y 4 B, C r y 10 A, \text { and } \\
C r y 11 A, C r y 30 A a \text { and Cry } \\
44 A a\end{array}$ & Leaf & $24^{\circ} 32^{\prime} \mathrm{N} 92^{\circ} 21^{\prime} \mathrm{E}$ & & Lepidoptera + Diptera \\
\hline TrBt-9 & $\begin{array}{l}\text { Bipyramidal } \\
\text { crystal }\end{array}$ & Cry $4 B$, Cry $12 A$ and Vip3A & Leaf & $24^{\circ} 32^{\prime} \mathrm{N} 92^{\circ} 21^{\prime} \mathrm{E}$ & & Lepidoptera + Diptera \\
\hline TrBt-10 & $\begin{array}{c}\text { Bipyramidal + } \\
\text { spherical }\end{array}$ & $\begin{array}{c}\operatorname{Cry} 2 A, C r y 3 A, C r y 8 A, C r y 11 A \\
\text { and Vip3A }\end{array}$ & $\begin{array}{l}\text { Dead } \\
\text { larvae }\end{array}$ & $24^{\circ} 32^{\prime} \mathrm{N} 92^{\circ} 21^{\prime} \mathrm{E}$ & $\begin{array}{l}\text { KC416619 } \\
\text { KC596017 } \\
\end{array}$ & Coleoptera \\
\hline AsBt-11 & $\begin{array}{l}\text { Bipyramidal }+ \\
\text { irregular }\end{array}$ & Cry1Ac, Cry16A, Vip3A & Leaf & $24^{\circ} 32^{\prime} \mathrm{N} 92^{\circ} 21^{\prime} \mathrm{E}$ & & Lepidoptera + Diptera \\
\hline AsBt-12 & $\begin{array}{c}\text { Bipyramidal } \\
\text { crystal }\end{array}$ & $\begin{array}{c}\text { CrylAc, Cry } 4 B, C r y 11 A, C r y 16 A \\
\text { and Vip3A }\end{array}$ & Leaf & $24^{\circ} 32^{\prime} \mathrm{N} 92^{\circ} 21^{\prime} \mathrm{E}$ & KC596015 & Lepidoptera+ Diptera \\
\hline AsBt-13 & $\begin{array}{c}\text { Bipyramidal }+ \\
\text { spherical }\end{array}$ & CrylAc and Vip3A & Leaf & $26^{\circ} 18^{\prime} \mathrm{N} 91^{\circ} 16^{\prime} \mathrm{E}$ & & Lepidoptera \\
\hline AsBt-14 & $\begin{array}{l}\text { Bipyramidal }+ \\
\text { Rhomboidal }+ \\
\text { Flat } \\
\end{array}$ & No amplification & Leaf & $26^{\circ} 18^{\prime} \mathrm{N} 91^{\circ} 16^{\prime} \mathrm{E}$ & & \\
\hline AsBt-15 & Bipyramidal & Cry $2 A$, Cry $12 A$, and Vip3A & Leaf & $25^{\circ} 42^{\prime} \mathrm{N} 88^{\circ} 24^{\prime} \mathrm{E}$ & KC596008 & Lepidoptera + Diptera \\
\hline AsBt-16 & $\begin{array}{l}\text { Bipyramidal + } \\
\text { Rhomboidal }\end{array}$ & $\begin{array}{c}\text { Cry } 2 A, C r y 4 A, C r y 7 A, C r y 8 A, \\
C r y 10 A, C r y 12 A, C r y 19 A \text { and } \\
\text { Vip3A }\end{array}$ & $\begin{array}{l}\text { Dead } \\
\text { larvae }\end{array}$ & $26^{\circ} 18^{\prime} \mathrm{N} 91^{\circ} 16^{\prime} \mathrm{E}$ & & Lepidoptera + Diptera \\
\hline TrBt-17 & Bipyramidal & $\begin{array}{c}C r y 2 A, C r y 3 A, C r y 7 A, C r y 8 A, \\
C r y 10 A, C r y 12 A, C r y 16 A \text { and } \\
\text { Vip3A }\end{array}$ & Soil & $23^{\circ} 51^{\prime} \mathrm{N} 91^{\circ} 16^{\prime} \mathrm{E}$ & KC416620 & Coleoptera \\
\hline TrBt-18 & $\begin{array}{l}\text { Bipyramidal } \\
+ \text { spherical }+ \\
\text { cuboidal }\end{array}$ & $\begin{array}{c}\text { Cry } 2 A, C r y 4 A, C r y 7 A, C r y 8 A \\
, C r y \text { 16A, and Vip3A }\end{array}$ & Leaf & $23^{\circ} 51^{\prime} \mathrm{N} 91^{\circ} 16^{\prime} \mathrm{E}$ & & $\begin{array}{c}\text { Lepidoptera }+ \text { Diptera }+ \\
\text { Coleoptera }\end{array}$ \\
\hline TrBt-19 & Bipyramidal & Cry $4 A$ and Vip3A & Leaf & $23^{\circ} 51^{\prime} \mathrm{N} 91^{\circ} 16^{\prime} \mathrm{E}$ & KC596007 & Lepidoptera + Diptera \\
\hline AsBt-20 & $\begin{array}{l}\text { Sphaerical }+ \\
\text { cuboidal }\end{array}$ & $\begin{array}{c}\text { Cry } 2 A, C r y 3 A, C r y 7 A, C r y 8 A, \\
\text { Cry } 10 A, C r y 12 A, C r y 16 A \text { and } \\
\text { Vip } 3 A\end{array}$ & Leaf & $26^{\circ} 18^{\prime} \mathrm{N} 91^{\circ} 16^{\prime} \mathrm{E}$ & KC416622 & Coleoptera \\
\hline AsBt-21 & $\begin{array}{l}\text { Sphaerical }+ \\
\text { cuboidal }\end{array}$ & $\begin{array}{c}\text { Cry } 2 A, C r y 3 A, C r y 7 A, C r y 8 A, \\
\text { Cry0A, Cry } 12 A \text { and Vip } 3 \mathrm{~A}\end{array}$ & Leaf & $26^{\circ} 18^{\prime} \mathrm{N} 91^{\circ} 16^{\prime} \mathrm{E}$ & KC416621 & Coleoptera \\
\hline
\end{tabular}




\begin{tabular}{|c|c|c|c|c|c|c|}
\hline AsBt-22 & Bipyramidal & Cryl6A & Leaf & $26^{\circ} 18^{\prime} \mathrm{N} 91^{\circ} 76^{\prime} \mathrm{E}$ & & Diptera \\
\hline AsBt-23 & Bipyramidal & Cryl6A & Soil & $22^{\circ} 70^{\prime} \mathrm{N} 78^{\circ} 20^{\prime} \mathrm{E}$ & & Diptera \\
\hline AsBt-24 & $\begin{array}{l}\text { Spherical + } \\
\text { cuboidal }\end{array}$ & $\operatorname{Cry} 3 A, \operatorname{Cry} 8 A$ and $C r y 12 A$ & Soil & $24^{\circ} 30^{\prime} \mathrm{N} 91^{\circ} 73^{\prime} \mathrm{E}$ & KC416623 & Diptera + Coleoptera \\
\hline AsBt-25 & $\begin{array}{l}\text { Bipyramidal } \\
+ \text { Rhomboidal }\end{array}$ & $\begin{array}{c}\operatorname{Cry} 2 A, \operatorname{Cry} 4 A, C r y 16 A \text { and } \\
\text { Vip } 3 \mathrm{~A}\end{array}$ & Soil & $24^{\circ} 30^{\prime} \mathrm{N} 91^{\circ} 73^{\prime} \mathrm{E}$ & KC596011 & Lepidoptera + Diptera \\
\hline AsBt-26 & Bipyramidal & No amplification & Soil & $24^{\circ} 30^{\prime} \mathrm{N} 91^{\circ} 73^{\prime} \mathrm{E}$ & & \\
\hline AsBt-27 & Bipyramidal & No amplification & Soil & $24^{\circ} 30^{\prime} \mathrm{N} 91^{\circ} 73^{\prime} \mathrm{E}$ & & \\
\hline AsBt-28 & Bipyramidal & No amplification & Soil & $24^{\circ} 30^{\prime} \mathrm{N} 91^{\circ} 73^{\prime} \mathrm{E}$ & & \\
\hline $\begin{array}{l}\text { Bt- } \\
\text { Assam }\end{array}$ & Bipyramidal & Cry $10 A$ and Cry $17 A$ & Soil & $26^{\circ} 18^{\prime} \mathrm{N} 91^{\circ} 16^{\prime} \mathrm{E}$ & & Diptera \\
\hline NE-60 & Bipyramidal & Cry 4A, Cry30Aa and Vip3A & Soil & $26^{\circ} 18^{\prime} \mathrm{N} 91^{\circ} 16^{\prime} \mathrm{E}$ & KC596010 & Lepidoptera + Diptera \\
\hline
\end{tabular}

Table 3. PCR analysis of cry genes occurring in the Bacillus thuringiensis isolates from North east

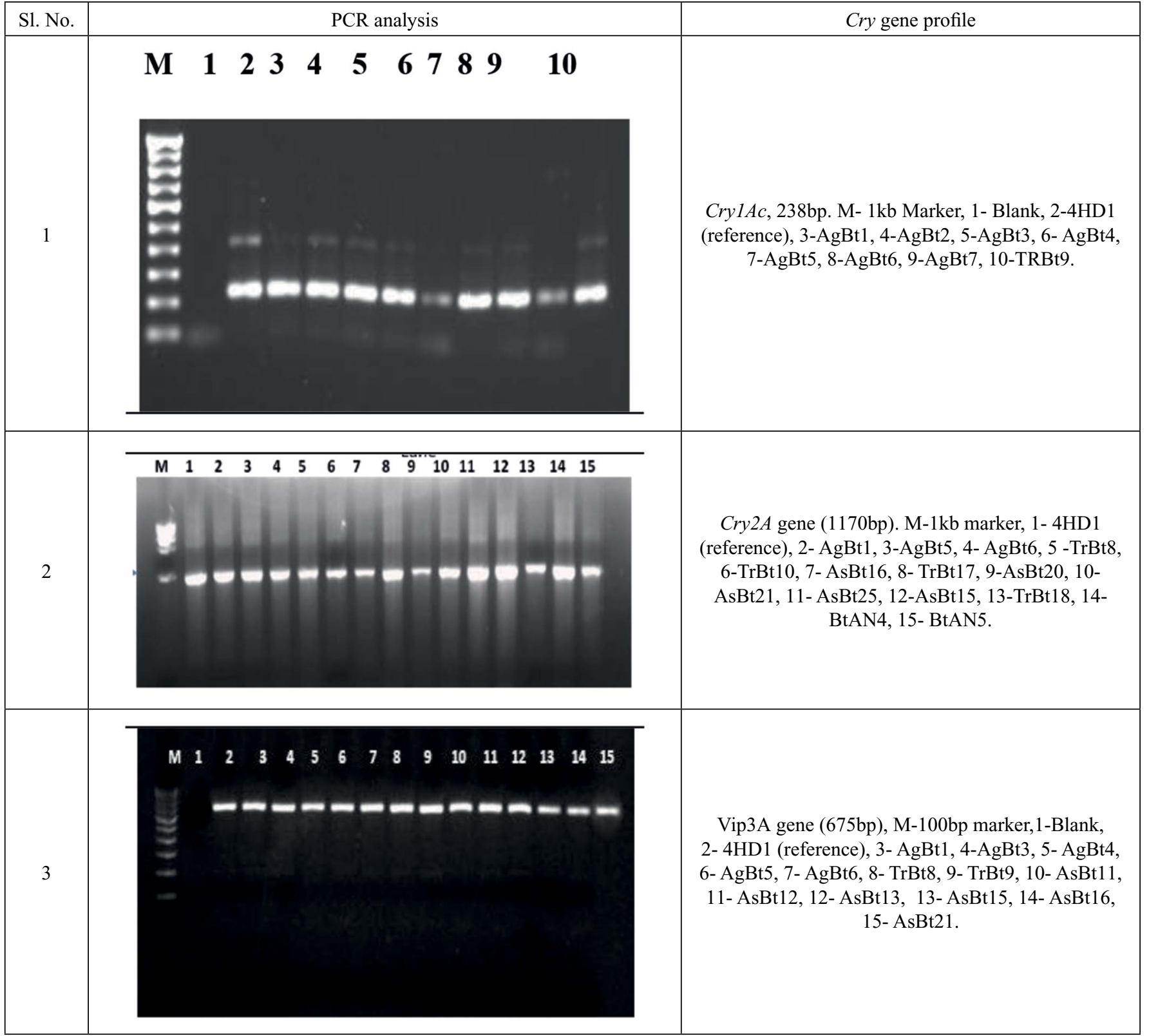




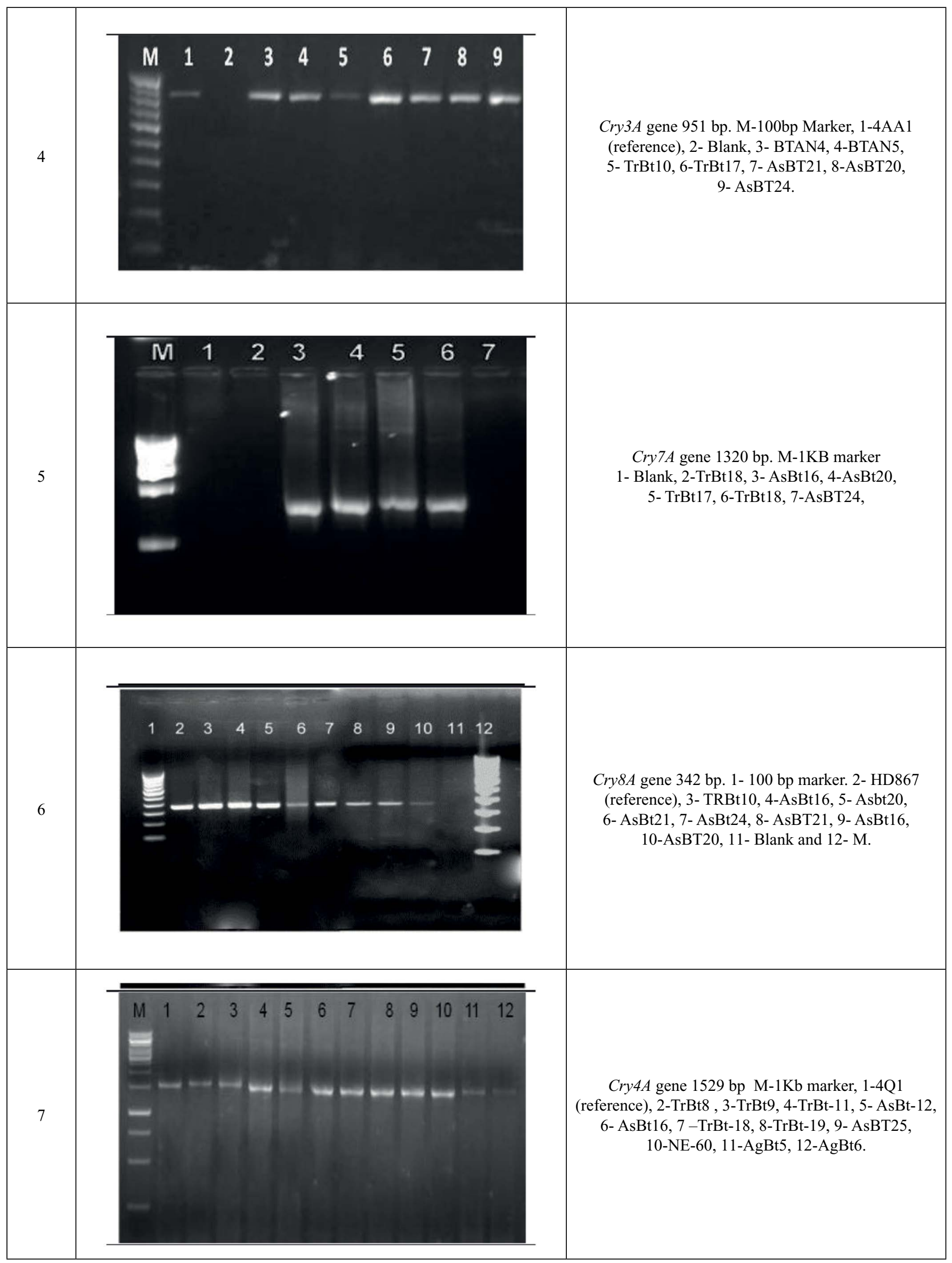




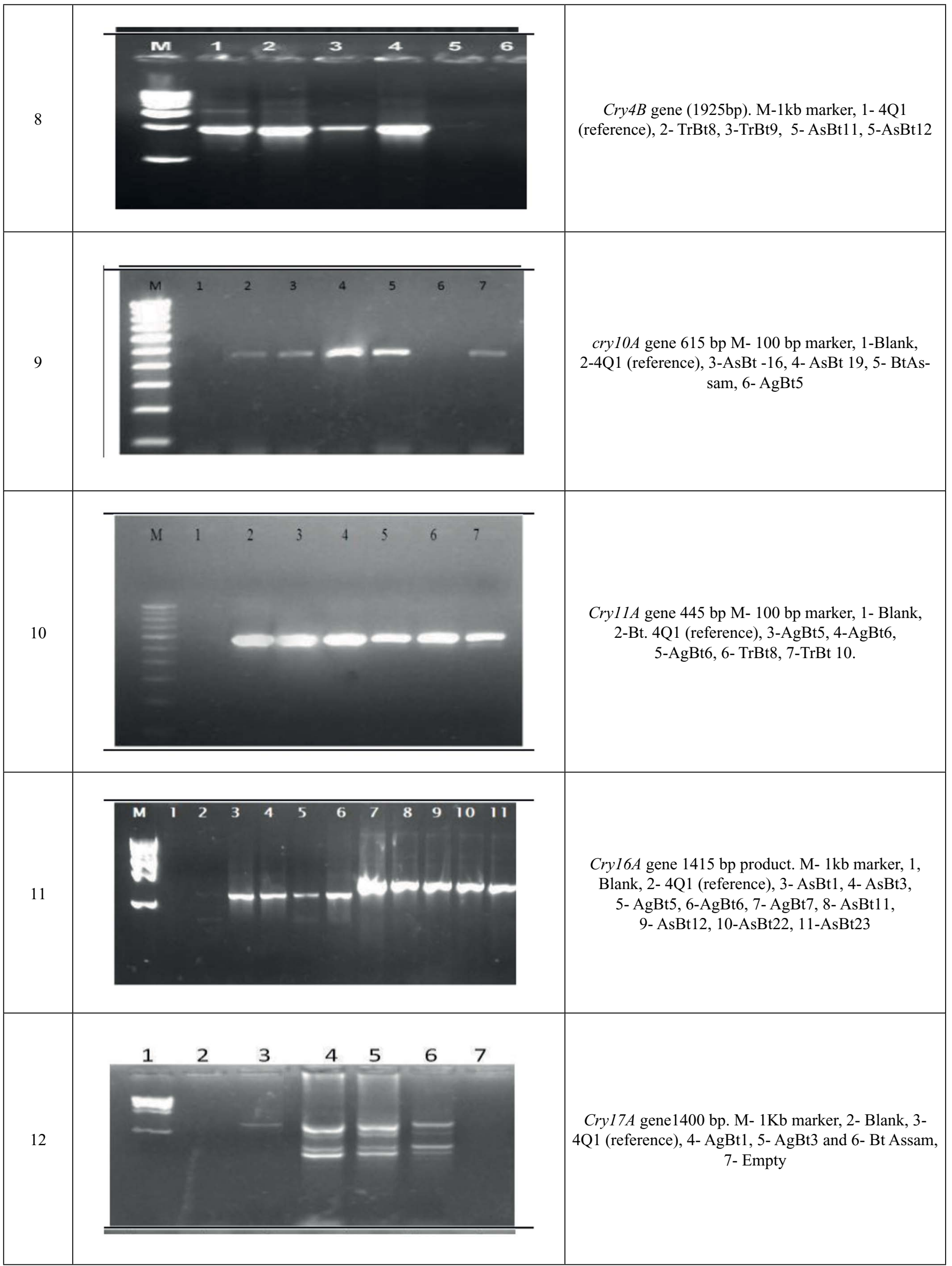




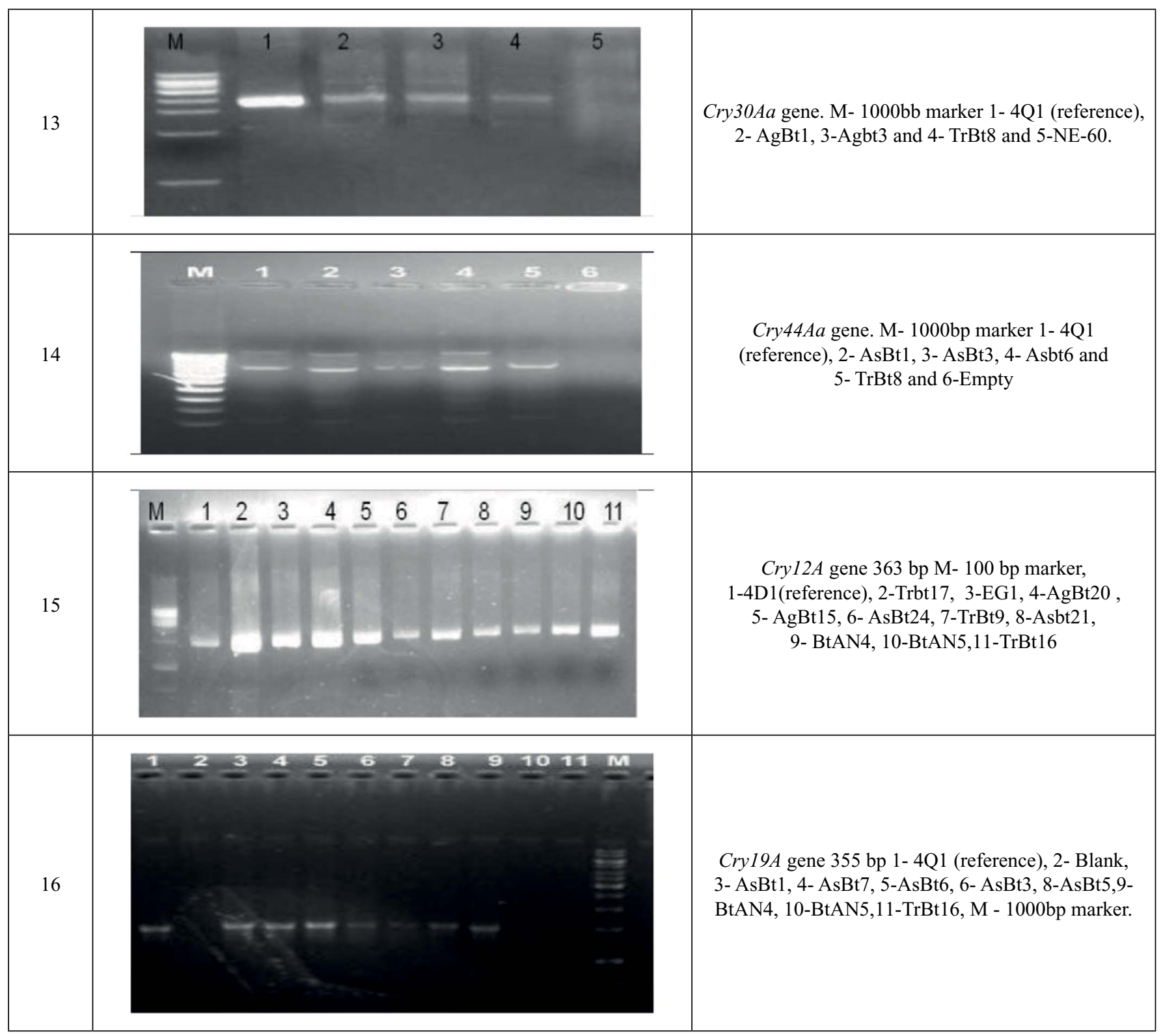

which could be active against Lepidoptera/Diptera/Coleoptera group of insects. The relative abundance of the cry genes in Northeast was analysed (Fig. 2). Vip3A was encountered in $16 \%$ of the samples, Cry $16 \mathrm{~A}$ was detected in $11 \%$ samples, $10 \%$ of specimens showed $C r y 1 A c$ and $C r y 2 A, C r y 10 A$ were $10 \%, C r y 12 A$ and $C r y 30 A$ was $6 \%, C r y 11 A, C r y 12 A$, Cry $8 A$ and $C r y 7 A$ was $4-5 \%$ and the other Cry protein genes ranged from $2-3 \%$. The cry toxins that are primarily active against lepidopteran insects belong to the Cryl, Cry2 and Vip3A groups. Cry3, Cry7, and $C r y 8$ toxins are active against coleopteran insects. The Cry $2 A$, Cry $4 A$, Cry $4 B$ Cry10A, Cry11A, Cry12A, Cry16A, Cry17A, Cry19A, Cry30Aa, and Cry $44 A$ a proteins are act against insects coming under order Diptera. Cry genes were amplified using specific primers and also reference strains. Cry gene profiling using specific or degenerate primers have been previously described (Ben Dov et al., 1997; 2001, Bravo et al., 1998, Ejiofor, 2002; Aly, 2007). Cry gene profile based on Lepidoptera encode for insect toxins namely cry1, cry2 and cry 9A whereas cry7 and cry8 encode for Coleopteran toxic proteins (Porcar and Perez, 2003, Ito et al., 2006). Konecka et al. (2012) analyzed the cry gene profile of 8 isolates and that they expressed diverse crystals and harboured cry genes active against pests of Coleoptera, Diptera and Lepidoptera. They could detect multiple cry genes like CrylAa, CrylAb, CrylAc, CrylB,

Cry1C, Cry1D, Cry1I, Cry2Aa, Cry2Ab, Cry9B, Cry9E, and Cry15. Cryl6A and Cry17A were first amplified in certain species of Clostridium (Barloy et al., 1996) and the gene products, Cryl6A and Cry17A, showed a remarkable 
Table 4. Bioassay of Bacillus thuringiensis samples expressing cry3a protein against Sitophilus oryzae

\begin{tabular}{|c|c|c|c|c|}
\hline \multirow{2}{*}{ Isolate } & \multirow{2}{*}{$\begin{array}{c}\text { LC50 } \\
\text { value } \\
\end{array}$} & \multicolumn{2}{|c|}{$95 \%$ confidence limits } & \multirow{2}{*}{ Std. Error } \\
\cline { 3 - 4 } & & Lower & Upper & \\
\hline 4AA1 & 27.129 & 15.736 & 35.792 & 0.51 \\
\hline AgBT 6 & 8.049 & 0.425 & 17.725 & 0.49 \\
\hline TrBT 17 & 13.893 & 1.894 & 23.624 & 0.54 \\
\hline AsBT 20 & 21.826 & 8.699 & 31.286 & 0.51 \\
\hline TrBT 10 & 19.122 & 1.842 & 31.638 & 0.48 \\
\hline AsBT 24 & 36.381 & 11.619 & 54.773 & 0.46 \\
\hline
\end{tabular}

Table 5. Bioassay of Bacillus thuringiensis samples expressing cry3a protein against Callosobrochus chinensis

\begin{tabular}{|c|c|c|c|c|}
\hline \multirow{2}{*}{ Isolate } & \multirow{2}{*}{$\begin{array}{c}\text { LC50 } \\
\text { value } \\
\end{array}$} & \multicolumn{2}{|c|}{$95 \%$ confidence limits } & \multirow{2}{*}{ Std. Error } \\
\cline { 3 - 4 } & Lower & Upper & \\
\hline $\begin{array}{c}\text { 4AA1 } \\
\text { (Standard })\end{array}$ & 15.963 & 4.579 & 24.487 & 0.56 \\
\hline AgBT 6 & 8.371 & 0.042 & 18.403 & 0.57 \\
\hline TrBT 17 & 40.928 & 21.738 & 58.298 & 0.47 \\
\hline AsBT 20 & 8.431 & 0.000 & 21.026 & 0.55 \\
\hline TrBT 10 & 15.898 & 4.219 & 24.795 & 0.54 \\
\hline AsBT 24 & 13.312 & 2.107 & 22.366 & 0.57 \\
\hline
\end{tabular}

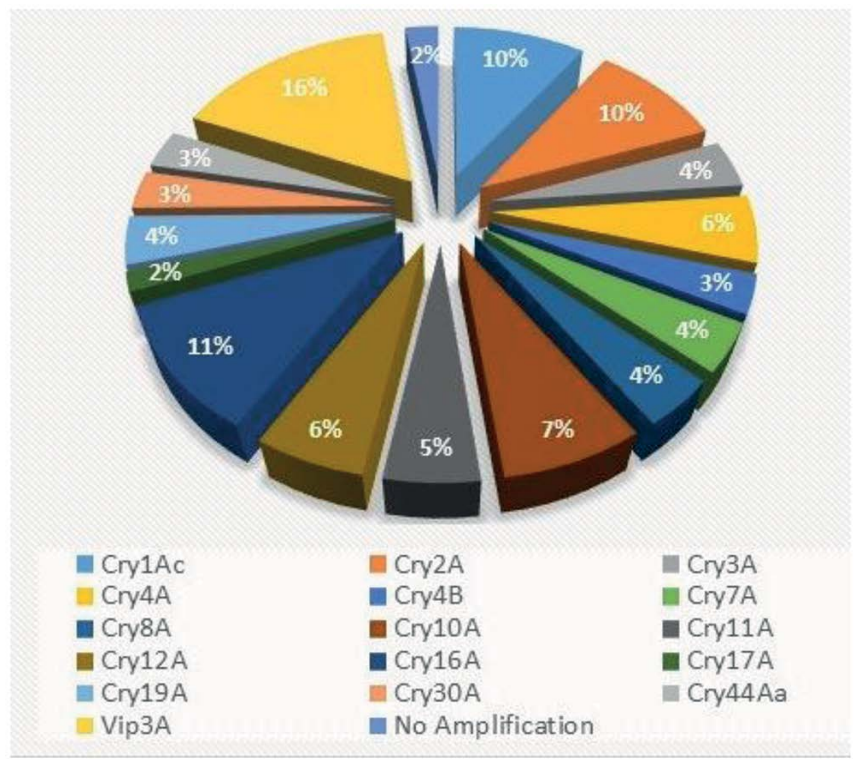

Fig. 1. Diversity of cry genes occurring in the North East.

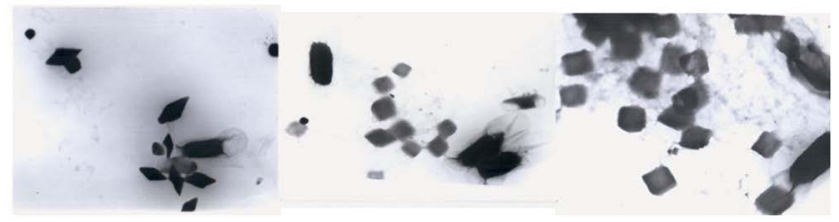

Fig. 2. Crystal morphology imaged through Transmission Electron Microscope (TEM).

mosquitocidal activity, and are the first reported cases of secreted or excreted mosquitocidal toxins derived from an anaerobic bacterium. In our studies we could amplify Cry $17 \mathrm{~A}$ in 3 isolates namely Ag-Bt1, Ag-Bt3 and Bt-Assam (Fig. 13) surprisingly Cryl6A was detected in 10 of the isolates (Fig. 12 and Table 3) based on size $1415 \mathrm{~kb}$ and 1400 $\mathrm{kb}$ gel picture. Nucleotide sequence BLAST analysis showed $99 \%$ similarity with the available database at NCBI. However further work on their cloning, sequence and bioassay needs to be studied. Ito et al. (2006), first reported the cloning and expression of two novel crystal protein genes, Cry44Aa/orf2$44 A$ and $C r y 30 B a / o r f 2-30 B$, from highly mosquitocidal $B$. thuringiensis subsp. entomocidus INA288. The Cry44Aa/ orf2-44A gene is highly toxic to $C$. pipiens and $A$. aegypti and appears to be a promising alternative to $B$. thuringiensis subsp. israelensis or may be used in combination with $B$. thuringiensis subsp. israelensis toxins. In the present study we detected these two rare cry genes in some of the isolates that showed presence of dipteran toxic cry proteins. Wild strains isolated from environmental samples can synthesize crystals that display higher activity against insect pests in comparison to $B$. thuringiensis strains already used in pesticide production (Konecka et al., 2012). The knowledge on coding for genes toxins in crystalline inclusion is useful in predicting potential pathogenicity of $B$. thuringiensis isolates against insects (Baig and Mehnaz, 2010; Nazarian et al., 2009).

Since some of the isolated $B t$ had Coleoptera specific cry genes, they were evaluated against Callosobrochus chinensis and $S$. oryzae. The results showed that the Agartala isolate NBAIR-AgBt6 was most toxic recording $\mathrm{LC}_{50}$ of $8.049 \mu \mathrm{g} / \mathrm{ml}$ against $S$. oryzae and $8.371 \mu \mathrm{g} / \mathrm{ml}$ against $C$. chinensis (Table 4 and 5). The isolate could be safer alternative to chemicals especially for stored grains. Stored grain pests cause heavy loss to granaries all over the world and accounts for 10 to 40 per cent loss (Chaubey, 2011). The pests mostly belong to the order Coleoptera. In India the main stored grain pests include C. chinensis, Sitophilus spp. and Tribolium spp. Strain HD73 was found to be the most active strain against $C$. chinensis that could be due to the variability of delta-endotoxins of different subspecies of these bacilli (Federiei, 1990). Asokan et al. (2013) obtained 36 new $B t$ isolates from Andaman and Nicobar islands and found variations in crystal morphology 
and mass of crystal protein(s). Based on the toxicity test, 50 $\%$ of isolates were toxic to ash weevils (coleopteran pest) and PCR analysis unveiled prepotency of $c r y l B$ and $c r y 8 b$ like genes in these isolates.

The results showed that dipteran and lepidopteran specific genes were predominant. In all the studies reference strains B. thuringiensis subsp. kurstaki (HD-1), B. thuringiensis subsp. israelensis, (4Q1), B. thuringiensis subsp. tenebrionis and $B$. thuringiensis subsp. japonensis were used for PCR standardization. Presence of Cry $16 A$ and $C r y 17 A$ is a first report for $B$. thuringiensis isolates from soils of North East.

\section{REFERENCE}

Aly AH Nariman. 2007. PCR Detection of cry genes in local Bacillus thuringiensis Isolates. Aust J Basic Appl Sci. 1(4): 461-466.

Aronson AI. 1994. Bacillus thuringiensis and its use as biological insecticide. Plant Breed Rev. 12: 19-45.

Asokan R, Mahadeva Swamy HM, Birah A, Geetha G Thimmegowda. 2013. Bacillus thuringiensis Isolates from Great Nicobar Islands. Curr Microbiol. 66: 621626.

Baig DN, Mehnaz S. 2010. Determination and distribution of cry-type genes in halophilc Bacillus thuringiensis isolates of Arabian Sea sedimentary rocks. Microbiol Res. 165(5):376-83.https://doi.org/10.1016/j.micres.2009.08.003 PMid:19850456

Baig DN, Bukhari DA, Shakoori AR. 2010. Cry genes profiling and the toxicity of isolates of Bacillus thuringiensis from soil samples against American bollworm, Helicoverpa armigera. J Appl Microbiol. 109(6): 1967-1978. https://doi.org/10.1111/j.13652672.2010.04826.x PMid:20738439

Barloy F, Lecadet M-M, Delécluse A. 1998. Distribution of clostridial cry-like genes among Bacillus thuringiensis and Clostridium strains. Curr Microbiol. 36: 232-237. https://doi.org/10.1007/s002849900300 PMid:9504991

Ben-Dov E, Zaritsky A, Dahan E, Barak Z, Sinai R, Manasherob R, A Khamraev, E Troitskaya, Dubitsky A, Berezina N, Margalith Y. 1997. Extended screening by PCR for seven cry-group genes from field-collected strains of Bacillus thuringiensis. Appl Environ Microbiol. 63: 4883-90.

Bourque SN, Valero JR, Mercier J, Lavoie MC, Levesque RC. 1993. Multiplex polymerase chain reaction for detection and differentiation of the microbial insecticide Bacillus thuringiensis. Appl Environ Microbiol. 59:(2): 523-527

Bravo A, Sarabia S, Lopez L, Ontiveros H, Abarca C, Ortiz A, Soberon M, Quintero, R. 1998. Characterization of cry genes in a Mexican Bacillus thuringiensis strain collection. Appl Environ Microbiol. 64: 4965-4972.

Chaubey MK. 2011. Combinatorial action of essential oils towards pulse beetle Callosobruchus chinensis Fabricius (Coleoptera: Bruchidae). Int J Agri Res. 6: 511-516. https://doi.org/10.3923/ijar.2011.511.516

Chen ML, Chen PH, Pang JC, Chia-Wei Lin CW, Chin-Fa HC, Hau-Yang T. 2014. The correlation of the presence and expression levels of cry genes with the insecticidal activitiesagainst Plutella xylostella for Bacillus thuringiensis strains. Toxins 6: 2453-2470. https://doi.org/10.3390/toxins6082453 PMid:25153253 PMCid:PMC4147593

Ejiofor AO, Johnson T. 2002. Physiological and molecular detection of crystalliferous Bacillus thuringiensis strains from habitats in the South Central United States. J Ind Microbiol Biotechnol. 28: 284-290 https://doi. org/10.1038/sj/jim/7000244 PMid:11986933

Federiei BA, Luthy P, Ibarra JE. 1990. Parasporal body of Bacillus thuringiensis israelensis: Structure, protein composition and toxicity. p. 349. In: de Barjac $\mathrm{H}$ and Sutherland DJ (Eds.). Bacterial control of mosquitoes and black flies. New Brunswick, Rutgers University Press. https://doi.org/10.1007/978-94-011-5967-8_3

Head G. 2005. Assessing the influence of Bt crops on natural enemies. Second Inter. Symp. on Biol. Control of Arthropods. Davos, Switzerland, Sept., 12-16.

Hofte H, Whiteley HR. 1989. Insecticidal crystal proteins of Bacillus thuringiensis. Microbiol. Rev. 53: 242-255.

Ito T, Ikeya T, Sahara K, Bando H. and Shin-ichiro A. 2006. Cloning and expression of two crystal protein genes, Cry30Bal and Cry44Aal, obtained from a highly mosquitocidal strain, Bacillus thuringiensis subsp. entomocidus. Appl Environ Microbiol. 72:(8): 5673-5676. https://doi.org/10.1128/AEM.01894-05 PMid:16885329 PMCid:PMC1538732

Konecka E, Baranek J, Hrycak A, Kaznowski A. 2012. Insecticidal activity of Bacillus thuringiensis Strains isolated from soil and water. Scientific World J. 2012: $1-5$. 
Lowry OH, Rosebrough NJ, Farr AL, Randall RJ. 1951. Protein measurement with the folin phenol reagent. $J$ Biol Chem. 193: 265-75.

Md. Abdur Rashid, Bhuiyan Al Sazzad, Rowshan Ara Begum, Reza Md. Shahjahan. 2012. Mortality effect of $B t$ extracts and esterase variability in three stored grain insects: Callosobruchus chinensis, Sitophilus granarius and Tribolium castaneum. Int J Agric Food Sci. 2(4) : 158-163.

Morris ON, Converse V, Kanagaratnam P, Cote JC. 1998. Isolation, characterization, and culture of Bacillus thuringiensis from soil and dust from grain storage bins and their toxicity for Mamestra configurata (Lepidoptera: Noctuidae). Can Entomol. 130: 515-537. https://doi.org/10.4039/Ent130515-4

Nazarian A, Jahangiri R, Jouzani GS, Seifinejad A, Soheilivand S, Bagheri O, Keshavarzi M, Alamisaeid K. 2009. Coleopteran-specific and putative novel cry genes in Iranian native Bacillus thuringiensis collection. J Invertebr Pathol. 102: 101-109. https://doi. org/10.1016/j.jip.2009.07.009 PMid:19631215

Porcar M, Juarez-Perez VP. 2003. PCR-based identification of Bacillus thuringiensis pesticidal crystal genes. FEMS Microbiol Rev. 26(5): 419-32. https:// doi.org/10.1111/j.1574-6976.2003.tb00624.x PMid:12586389

Quesada-Moraga E, Garcia-Tovar E, Valverde-Garcia P, Santiago-Alvarez C. 2004. Isolation, geographical diversity and insecticidal activity of Bacillus thuringiensis from soils in Spain. Microbiol Res. 159:(2004): 59-71. https://doi. org/10.1016/j.micres.2004.01.011 PMid:15160608

Ramalakshmi A, Udayasuriyan V. 2010. Diversity of Bacillus thuringiensis isolated from Western Ghats of Tamil Nadu State, India. Curr Microbiol. 61(1): 13-8. doi: 10.1007/ s00284-009-9569-6. https://doi.org/10.1007/s00284-0099569-6 PMid:20033169.

Rangeshwaran R, Velavan V, Frenita DL, Surabhi Kumari, Shylesha AN, Mohan M, Satendra Kumar and Sivakumar G. 2016. Cloning, expression and bioassay of Vip3A protein from an indigenous Bacillus thuringiensis isolate. J Pure Appl Microbiol. 10(2): 1533-1539

Salehi Jouzani G, Seifinejad A, Saeedizadeh A, Nazarian A, Yousefloo M, Soheilivand S, Mousivand M, Jahangiri R, Yazdani M, Amiri RM, Akbari S.2008. Molecular detection of nematicidal crystalliferous Bacillus thuringiensis strains of Iran and evaluation of their toxicity on free-living and plant-parasitic nematodes. Can J Microbiol. 54: 812-822. https://doi.org/10.1139/ W08-074 PMid:18923549

Sambrook J, Fritsch EF, Maniatis T. 1989. Molecular cloning: a laboratory manual. $2^{\text {nd }}$ edition. Cold Spring ndHarbor Laboratory, Cold Spring Harbor, N.Y.

Santana MA, Moccia-V CC, Gillis AE. 2008. Bacillus thuringiensis improved isolation methodology from soil samples. J Microbiol Methods 75:(2): 357-8. doi: 10.1016/j.mimet.2008.06.008. https://doi.org/10.1016/j. mimet.2008.06.008 PMid:18619500

Schnepf E, Crickmore N, Van Rie J, Lereclus D, Baum J, Feitelson J, Zeigler DR, Dean DH. 1998. Bacillus thuringiensis and its pesticidal crystal proteins. Microbiol Mol Biol Rev. 62: 775-806.

Thammasittirong A, Attathom T. 2008. PCR-based method for the detection of cry genes in local isolates of Bacillus thuringiensis from Thailand. J Invertebr Pathol. 98: 121-126. https://doi.org/10.1016/j.jip.2008.03.001 PMid:18407288

Travers RS, Martin PA, Reichelderfer CF. 1987. Selective process for efficient isolation of soil Bacillus spp. Appl Environ Microbiol. 53: 1263-1266.

Uribe D, Martinez W and Ceron J. 2003. Distribution and diversity of cry genes in native strains of Bacillus thuringiensis obtained from different ecosystems from Colombia. J Invertebr Pathol. 82: 119-127. https://doi. org/10.1016/S0022-2011(02)00195-7

Van Frankenhuyzen K (2009) Insecticidal activity of Bacillus thuringiensis crystal proteins. J Invertebr Pathol. 101: 1-16. https://doi.org/10.1016/j.jip.2009.02.009 PMid:19269294

Vidal-Quist JC, Castañera P. and González-Cabrera J. 2009. Diversity of Bacillus thuringiensis strains isolated from citrus orchards in Spain and evaluation of their insecticidal activity against Ceratitis capitata. J Microbiol Biotechnol. 19(8): 749-759.

Zothansanga, Lalhmachhuani N, Senthil Kumar N, Gurusubramanian G. 2011. PCR pathotyping of native Bacillus thuringiensis from Mizoram, India. Sci Vis. 11(3): 171-176. 